\title{
Differing clinical characteristics between influenza strains among young healthy adults in the tropics
}

Jonathan Yap ${ }^{1 \dagger}$, Chi Hsien Tan ${ }^{1 \dagger}$, Alex R Cook ${ }^{2,3+}$, Jin Phang Loh ${ }^{4}$, Paul A Tambyah ${ }^{5}$, Boon Huan $\operatorname{Tan}^{4}$ and Vernon J Lee Le, $^{1,7^{*}+}$

\begin{abstract}
Background: Influenza infections may result in different clinical presentations. This study aims to determine the clinical differences between circulating influenza strains in a young healthy adult population in the tropics.

Methods: A febrile respiratory illness (FRI) (fever $\geq 37.5^{\circ} \mathrm{C}$ with cough and/or sore throat) surveillance program was started in 4 large military camps in Singapore on May 2009. Personnel with FRI who visited the camp clinics from 11 May 2009 to 25 June 2010 were recruited. Nasal washes and interviewer-administered questionnaires on demographic information and clinical features were obtained from consenting participants. All personnel who tested positive for influenza were included in the study. Overall symptom load was quantified by counting the symptoms or signs, and differences between strains evaluated using linear models.
\end{abstract}

Results: There were 434 (52.9\%) pandemic H1N1-2009, 58 (7.1\%) seasonal H3N2, 269 (32.8\%) influenza B, and 10 (1.2\%) seasonal H1N1 cases. Few seasonal influenza A (H1N1) infections were detected and were therefore excluded from analyses, together with undetermined influenza subtypes (44 (1.5\%)), or more than 1 co-infecting subtype (6 (0.2\%)). Pandemic H1N1-2009 cases had significantly fewer symptoms or signs (mean 7.2, 95\%Cl 6.9-7.4, difference $1.6,95 \% \mathrm{Cl} 1.2-2.0, p<0.001$ ) than the other two subtypes (mean $8.7,95 \% \mathrm{Cl} 8.5-9.0$ ). There were no statistical differences between H3N2 and influenza B $(p=0.58)$. Those with nasal congestion, rash, eye symptoms, injected pharynx or fever were more likely to have H3N2; and those with sore throat, fever, injected pharynx or rhinorrhoea were more likely to have influenza B than H1N1-2009.

Conclusions: Influenza cases have different clinical presentations in the young adult population. Pandemic H1N1 influenza cases had fewer and milder clinical symptoms than seasonal influenza. As we only included febrile cases and had no information on the proportion of afebrile infections, further research is needed to confirm whether the relatively milder presentation of pandemic versus seasonal influenza infections applies to all infections or only febrile illnesses.

\section{Background}

Influenza infections arising from different influenza strains may result in different clinical presentations. Determining these different clinical presentations is useful for epidemiological comparison. Furthermore, having knowledge of such clinical symptoms may aid clinicians in determining shifts in influenza strains and managing selected patients at higher risk of developing complications from influenza, especially in settings with poor laboratory resources. Few studies have differentiated the

\footnotetext{
* Correspondence: vernonljm@hotmail.com

† Contributed equally

${ }^{1}$ Biodefence Centre, Ministry of Defence, Singapore

Full list of author information is available at the end of the article
}

clinical presentation of different influenza strains, particularly in the tropics where the spread of influenza differs from temperate regions [1]. One recent tropical study explored the differences in presentation among various influenza subtypes, but was based on limited number of hospital attendances during the onset of the 2009-H1N1 pandemic [2].

Despite earlier hypotheses that the H1N1-2009 pandemic strain would become the predominant influenza strain in circulation [3], the influenza A (H3N2) and influenza $B$ subtypes that were in circulation before the pandemic continue to circulate globally. This provides the opportunity to compare their clinical features. As older children and young adults were substantially 
affected by the H1N1-2009 outbreaks [4], this study uses a military respiratory disease surveillance program in tropical Singapore to determine the clinical differences between circulating influenza strains among young healthy adults presenting with febrile respiratory illness [5].

\section{Methods}

This study was carried out as part of a respiratory disease surveillance program within the Singapore military. The febrile respiratory illness (FRI) (fever $\geq 37.5^{\circ} \mathrm{C}$ with cough and/or sore throat) surveillance program was started in 4 large military camps in May 2009 before community spread of pandemic H1N1-2009 in Singapore [6]. All personnel with FRI who visited the camps' primary healthcare clinics from 11 May 2009 to 25 June 2010 were recruited into the study - this study therefore excludes milder cases, and compares the different clinical symptoms among FRI cases with influenza. Nasal washes from each side of the nose were taken from consenting participants by trained medical staff, placed in viral transport media, and sent to the laboratory for aetiological testing within $24 \mathrm{~h}$. In addition, interviewer-administered questionnaires on demographic information and clinical features of infection were obtained during the clinical consultation, and again at 2 weeks post-consult via telephone interview to identify symptoms present during the entire illness course.

Written informed consent was obtained. Approval was given by the military's Joint Medical Committee for Research, and the respective institutional review boards of the National University of Singapore and Australian National University.

\section{Laboratory methods}

To determine aetiology of the FRI cases, we used the Resplex II (version 2.0, Qiagen, Inc., Valencia, CA, USA) multiplex PCR assay as previously described in other studies $[5,7,8]$. The Resplex II assay is a multiplex PCR assay coupled with bead array detection technology that is able to detect multiple viruses including influenza $\mathrm{A}$ and influenza $B[7,8]$. For each nasal wash specimen, total nucleic acids were extracted using the DNA minikit (Qiagen, Inc, Valencia, CA, USA) and $5 \mu$ of extract were tested with Resplex II on the LiquiChip 200 Workstation.

Specimens identified as Influenza A positive by the Resplex II assay underwent additional singleplex real-time PCR assays for H1, H3, or pandemic H1N1-2009 as previously described $[5,9]$. To determine the circulating lineages of influenza, selected specimens were partially sequenced by cDNA synthesis using the uni12primer (AGCAAAAGCAGG) [10] with Transcriptor First Strand cDNA synthesis Kit (Roche Diagnostics, Mannheim, Germany). PCR amplification of the partial H1 gene and H3 gene were carried out with primers described previously [11]. The amplicons were then sequenced using the primers and analysed with DNAstar, Lasergene version 7 (Madison, USA). For influenza B-positive specimens, partial HA gene PCR amplification was obtained with inhouse designed primers (HA471F $=$ 5'ACCTCAGGATCTTGCCCTAACG-3' and HA1169R = 5'TGTGTAT CCGTGCCAACCTGCAAT-3') and sequenced with the same primers as described for the sequencing of the influenza A strains above.

\section{Statistical analysis}

To fulfil the main aim of the study, we analyzed the overall clinical differences among pandemic influenza A (H1N1-2009), influenza A (H3N2), and influenza B. Few seasonal influenza A (H1N1) infections were detected and these were therefore excluded from analyses, along with influenza cases in which no subtype could be determined due to insufficient sample quantity (44 cases, $1.5 \%)$, and patients with multiple co-infecting subtypes (6 cases, $0.2 \%$ ).

We performed two forms of statistical analysis. The first conditions on influenza subtype and addresses the questions: given a patient is infected by this strain, how will his illness express itself in terms of individual symptoms (or signs), syndromes of pairs of symptoms, and overall symptom load, relative to other aetiologies? Overall symptom load was quantified by counting the symptoms or signs, and differences in overall symptom load between strains evaluated using linear models. Empirical proportions of clinical presentation were used to assess patterns, for each pair of strains, with logistic regression to evaluate differences in symptom expression. We evaluated the presence of paired syndromes by assessing whether the joint distributions of symptom pairs were more or less likely to occur together using binomial tests, defining the excess probability ratio to be the ratio of the proportion of cases in which the pair occurred together relative to the product of the marginal proportion of each symptom.

The second analysis conditions on the suite of symptoms enumerated in this study and asks: given that the patient has expressed this set of symptoms, what is the likely aetiology? In this analysis, potential confounding was addressed by multivariate analyses between pairs of strains, with preliminary models containing all statistically significant variables on univariate analysis across all subtypes, before eliminating non-significant symptom variables sequentially starting with the one with the highest $p$-value. No interactions between variables were significant.

Statistical analyses were performed using R [12], with a significance level of 0.05 .

\section{Results}

A total of 2858 participants ( $98 \%$ of those eligible) were recruited during the study period, with 2717 (95.1\%) 
participants completing the follow-up survey. Participants were mostly young adults (average age 21 years, SD 3) and male (2853, 99.8\%) due to the demographics of the military setting. There were a total of 821 influenza cases - of which 434 (52.9\%) were pandemic H1N1-2009, 58 (7.1\%) seasonal H3N2, 269 (32.8\%) influenza B, and $10(1.2 \%)$ seasonal H1N1. The influenza vaccination status of the influenza cases is presented in Table 1 . The circulating strains during the study period were the A/California/7/2009(H1N1)-like virus, A/Perth/ $16 / 2009$-(H3N2)-like virus, and influenza B from the Victoria-lineage, closely related to the B/Brisbane/60/ 2008-like virus. Figure 1 shows the distribution of influenza cases over the course of the study.

From the comparison among the three main influenza strains (pandemic H1N1-2009, seasonal H3N2, and influenza B), pandemic H1N1-2009 cases had significantly fewer symptoms or signs (mean 7.2, 95\%CI 6.9-7.4, difference 1.6, 95\%CI 1.2-2.0, $p<0.001$ ) than the other two subtypes (mean 8.7, 95\%CI 8.5-9.0). There were no statistical differences between $\mathrm{H} 3 \mathrm{~N} 2$ and influenza B $(p=0.58)$. The patterns of symptoms expressed also differed substantially between strains as shown in Figure 2. Pandemic H1N12009 cases were significantly less likely to have nasal congestion, eye or ear symptoms, chest pain, headache, high fever, or injected pharynx compared to H3N2; and less likely to have productive cough, sore throat, nasal congestion, rhinorrhoea, eye or ear symptoms, chills or rigors, myalgia or arthralgia, headache, high fever, or injected pharynx, but more likely to have dry cough compared to influenza B. H3N2 cases were associated with more dry cough and rash but less productive cough or sore throat than influenza B.

After adjusting for potential confounders as shown by the multivariate analysis in Table 2, cases with nasal congestion, rash, eye symptoms, injected pharynx or fever were more likely to be infected with H3N2 than pandemic H1N1-2009. Those with sore throat, fever, injected pharynx or rhinorrhoea were more likely to be infected with influenza B than H1N1-2009, while dry cough patients were more likely to be infected with H1N1-2009 than influenza B. Those with nasal congestion or rash were more likely to be infected with H3N2 than influenza B but those with productive cough were more likely to be

Table 1 Influenza vaccination status of influenza cases

\begin{tabular}{ccccc}
\hline Influenza cases & \multicolumn{4}{c}{ Type of influenza vaccine received } \\
\cline { 2 - 5 } & Nil & Pandemic vaccine & Seasonal vaccine & Both \\
\hline H1N1pdm & 345 & 33 & 46 & 10 \\
\hline H3N2 & 40 & 7 & 6 & 5 \\
\hline H1N1 & 10 & 0 & 0 & 0 \\
\hline B & 41 & 212 & 2 & 14 \\
\hline
\end{tabular}

infected with influenza B than H3N2. None of the subjects required hospitalisation.

Figure 3 shows that some symptoms were significantly associated (or dissociated) with other symptoms. Some are obvious, such as dry and productive coughs which were dissociated, while fever $\geq 37.8^{\circ} \mathrm{C}$ and fever $\geq 38^{\circ} \mathrm{C}$ were associated. For pandemic H1N1-2009, some less common symptom pairs appeared together more than twice as often as expected: chest pain was strongly associated with conjunctivitis, dyspnoea, diarrhoea and ear ache, while nausea or vomiting was strongly associated with diarrhoea and photophobia, and conjunctivitis with ear ache. Few patients presented with both diarrhoea and a dry cough. Many of the more common symptoms also appeared as complexes, with the appearance of one increasing the chances of the simultaneous expression of others. For influenza $B$, chest pain also more frequently occurred if accompanied by dyspnoea, nausea or vomiting, diarrhoea, or ear ache; diarrhoea was often coupled with nausea or vomiting; dyspnoea with ear ache; photophobia with conjunctivitis or abnormal findings on clinical examination of the lungs; while abnormal clinical lung findings more often expressed themselves jointly with a dry cough than expected by chance. There were too few $\mathrm{H} 3 \mathrm{~N} 2$ cases to identify significant syndromes.

\section{Discussion}

Determining the clinical characteristics between circulating influenza strains is important for epidemiological decision making such as control and surveillance strategies, and for clinicians to understand the symptom complexes for different strains for patient management. Our peripandemic period data shows the diversity of clinical presentations among influenza strains - it is important to note that our results may only apply to the specific strains that we studied, and may not apply to other strains in general. Pandemic H1N1-2009 cases presented with fewer symptoms overall compared to seasonal H3N2 or influenza $\mathrm{B}$ cases, in spite of the propensity for pandemic H1N1-2009 to affect older children and young adults [4]. This shows that although the extent of spread of the 2009 pandemic was substantial in this population, the clinical severity may have been lower than even seasonal influenza strains, resulting in a lower overall impact compared to previous pandemics. In addition, as influenza spreads quickly in closed environments such as the military, schools, or boarding facilities [13], knowing the clinical characteristics of existing influenza strains might help raise the suspicion of novel influenza strains if marked changes in clinical characteristics are noted, and to identify novel strains for rapid initiation of control measures.

Our study showed that pandemic H1N1 cases were less likely to present with high temperature, sore throat, rhinorrhoea and injected pharynx compared to influenza 


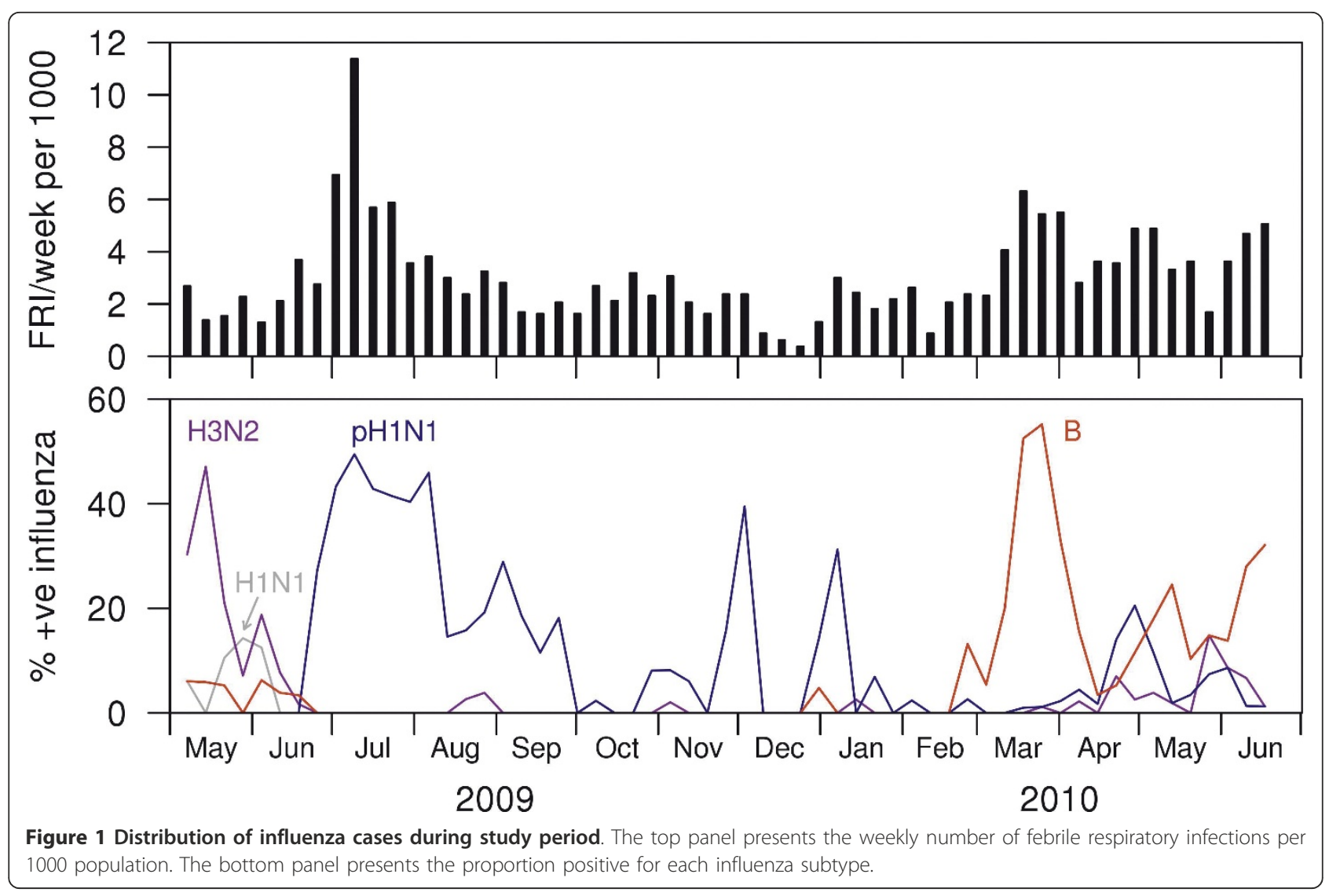

B cases; and less likely to present with high fever, rash, nasal congestion, eye symptoms and injected pharynx compared to H3N2 cases. A tropical study also found that pandemic H1N1-2009 presented with fewer symptoms than seasonal influenza; including less fever, rhinorrhoea and dyspnoea but more cough [2]. However, yet another found no differences in presentation between pandemic $\mathrm{H} 1 \mathrm{~N} 1$ and other influenza subtypes [14]. However, the latter two studies did not distinguish between various seasonal influenza subtypes. A study comparing adults with pandemic H1N1-2009 to those with H3N2 found no statistically significant differences for a smaller set of 12 symptoms [15]. In our study, subjects with nasal congestion or rash were more likely to be infected with H3N2 than influenza B but those with productive cough were more likely to be infected with influenza B. Another study from France showed that temperature of $>38.2^{\circ} \mathrm{C}$, stiffness or myalgia, rhinorrhoea, and cough were predictive of H3N2 infection, showing that differences exist for different strains in different settings [16].

Although reasons for different symptoms and syndromes caused by influenza subtypes are not immediately apparent, this may be related to host or viral factors which have yet to be elucidated. This should form the basis for further research into the pathogenic properties of influenza subtypes and strains; and the possible linkages to pre-existing immunity or cross-immunity from other strains.

The differences in the clinical symptoms from the influenza strains shown in this study also display the difficulty in using solitary descriptions of influenza (such as traditional ILI definitions) with predictable accuracy for clinical diagnosis and case management. The predictive accuracy for influenza in any setting will depend on the circulating influenza strains and other respiratory pathogens circulating locally. It is therefore important to combine clinical syndromic surveillance with effective laboratory testing. In the context of clinical management, more sophisticated rapid tests may be required to identify influenza cases with high sensitivity and specificity for clinical management and public health measures to be taken.

Our study's strengths are its large sample size, high follow-up rate, and high diagnostic ascertainment of influenza subtypes. Limitations include selection bias towards febrile symptomatic cases - although influenza may present as milder infections, these are difficult to identify through surveillance and we are concerned with more severe clinical presentations resulting in absenteeism. 


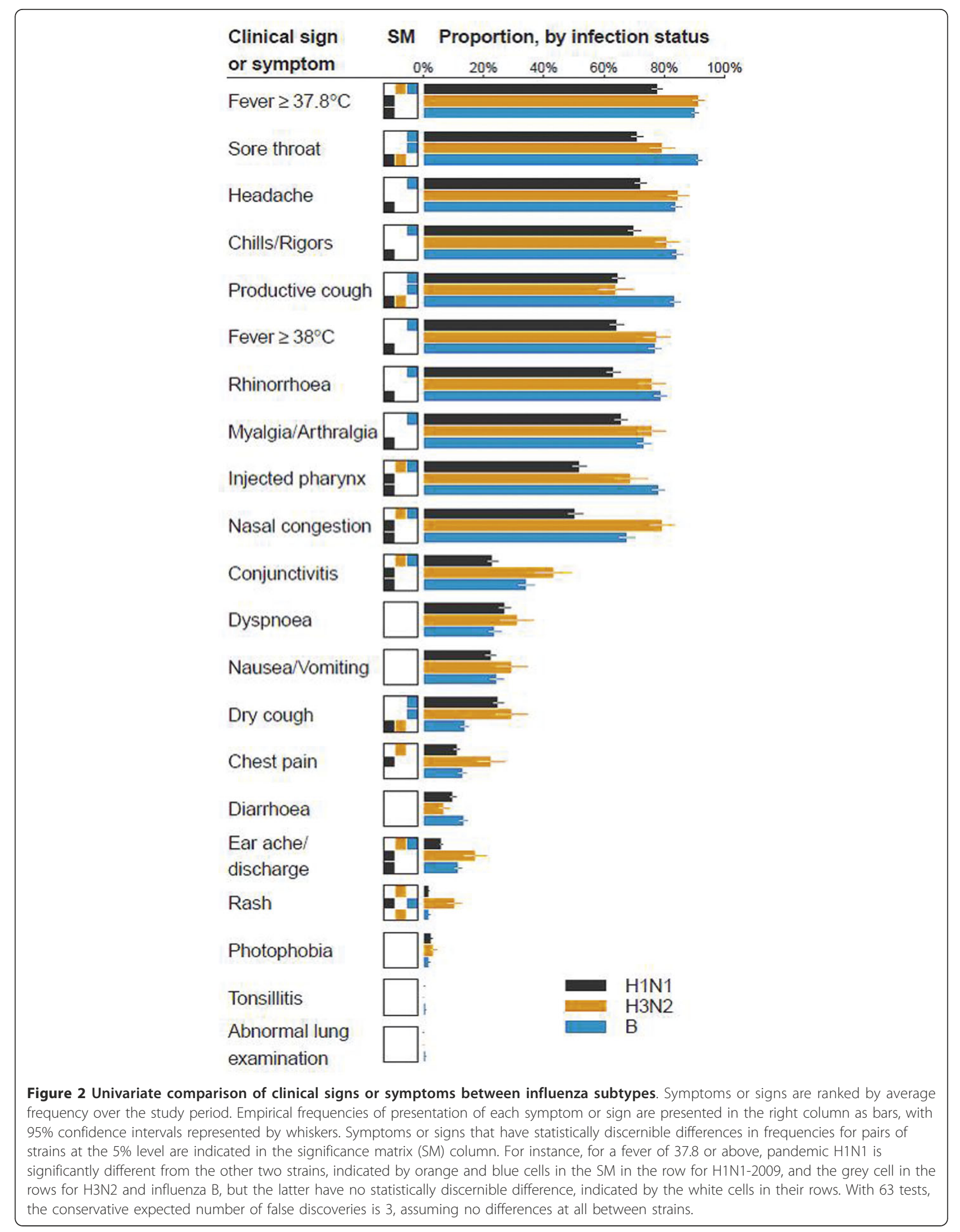


Table 2 Multivariate analysis comparing clinical features between influenza subtype- positive cases

\begin{tabular}{|c|c|c|}
\hline \multicolumn{3}{|c|}{ Influenza H1N1pdm vs H3N2* } \\
\hline Parameters & Adjusted odds ratio ( $95 \%$ Confidence interval) & pvalue \\
\hline Blocked nose & $0.33(0.16,0.72)$ & 0.005 \\
\hline Rash & $0.10(0.02,0.37)$ & $<0.001$ \\
\hline Eye symptoms & $0.47(0.24,0.93)$ & 0.03 \\
\hline Injected pharynx & $0.45(0.21,0.97)$ & 0.04 \\
\hline Fever $\left(\geq 37.8^{\circ} \mathrm{C}\right)$ & $0.33(0.11,0.99)$ & 0.05 \\
\hline \multicolumn{3}{|c|}{ Influenza H1N1pdm vs B* } \\
\hline Parameters & Adjusted odds ratio ( $95 \%$ Confidence interval) & $p$ value \\
\hline Sore throat & $0.44(0.24,0.80)$ & 0.007 \\
\hline Fever $\left(\geq 37.8^{\circ} \mathrm{C}\right)$ & $0.51(0.29,0.92)$ & 0.024 \\
\hline Injected pharynx & $0.45(0.29,0.72)$ & $<0.001$ \\
\hline Age (per year) & $1.25(1.08,1.46)$ & 0.003 \\
\hline Dry cough & $2.10(1.25,3.54)$ & 0.005 \\
\hline Running nose & $0.54(0.34,0.86)$ & 0.009 \\
\hline \multicolumn{3}{|c|}{ Influenza H3N2 vs B* } \\
\hline Parameters & Adjusted odds ratio ( $95 \%$ Confidence interval) & $p$ value \\
\hline Blocked nose & $3.00(1.24,7.19)$ & 0.01 \\
\hline Rash & $6.88(1.50,31.60)$ & 0.01 \\
\hline Age (per year) & $1.28(1.09,1.51)$ & 0.002 \\
\hline Cough with sputum & $0.24(1.11,0.51)$ & $<0.001$ \\
\hline
\end{tabular}

*Age, dry cough, wet cough, sore throat, running nose, blocked nose, sore eyes or eye pain, chills/rigors, myalgia/arthralgia, ear symptoms, rash, chest pain, headache, Fever $\geq 37.8^{\circ} \mathrm{C}$, Fever $\geq 38.0^{\circ} \mathrm{C}$, and injected pharynx were included in the analysis before non-significant terms were sequentially removed. With 48 tests, the conservative expected number of false discoveries is 2.4 .

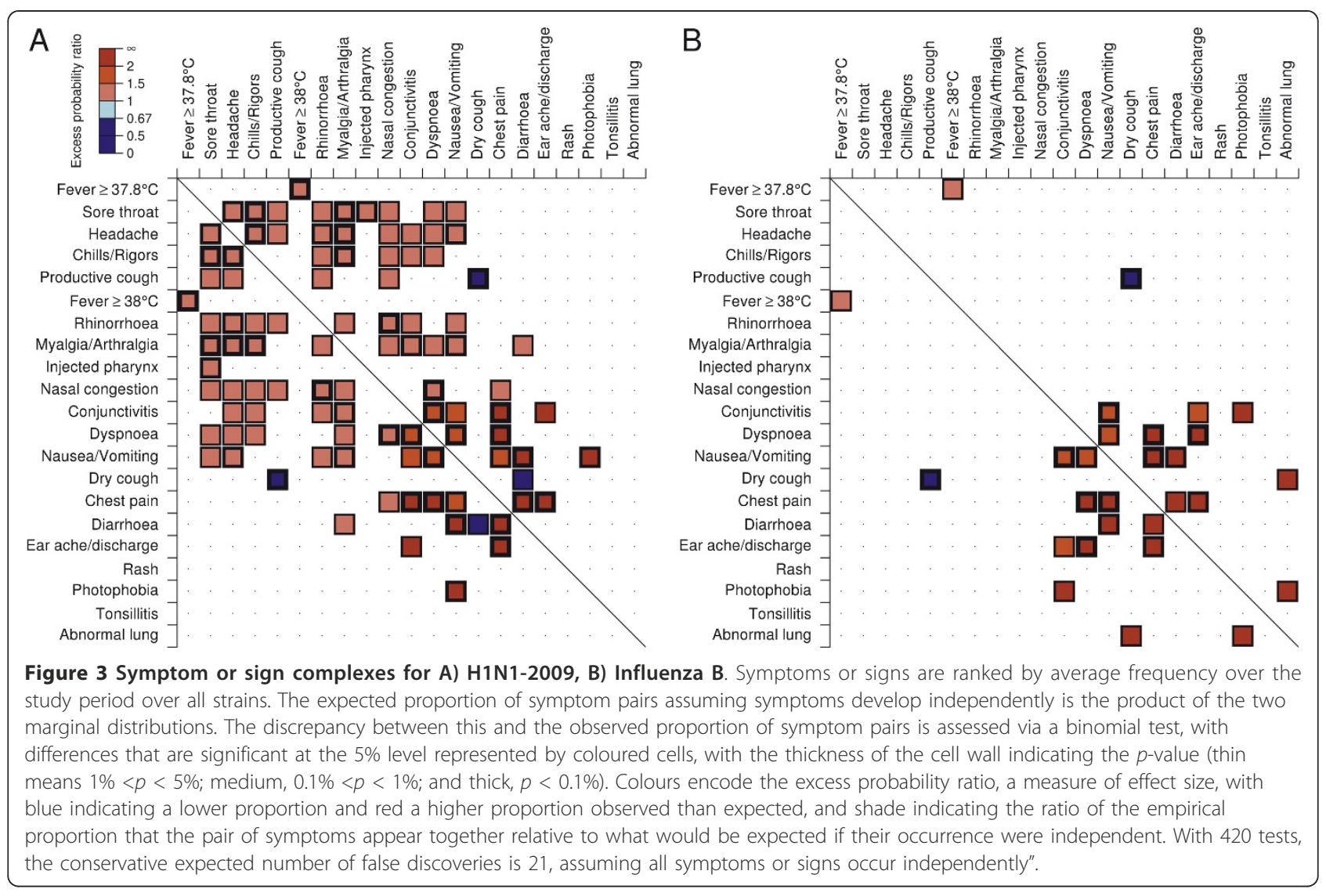


This study involved predominantly young adult males, and results may not be generalizable to the overall population, necessitating further studies to explore differences in presentation among various age groups and gender. Finally, the actual clinical impact of differentiating between various influenza subtypes may be limited and would depend on factors such as their current anti-viral resistance profiles. However, this is not examined in this study, and strains displaying anti-viral resistance may have different clinical presentations.

\section{Conclusion}

Different influenza strains have different clinical presentations in the young adult population, and pandemic H1N1 influenza cases had fewer and milder clinical symptoms than seasonal influenza H3N2 and B.

\section{Funding source}

The work was supported by a Singapore Ministry of Defence funded operational research program. The funders had no role in study design, data collection and analysis, decision to publish, or preparation of the manuscript.

\section{Author details \\ 'Biodefence Centre, Ministry of Defence, Singapore. ${ }^{2}$ Department of Statistics and Applied Probability, National University of Singapore, Singapore. ${ }^{3}$ Program in Health Services and Systems Research, Duke-NUS Graduate Medical School, Singapore. ${ }^{4}$ Defence Medical and Environmental Research Institute, DSO National Laboratories, Singapore. ${ }^{5}$ Department of Medicine, National University Health System, Singapore. ${ }^{6}$ Saw Swee Hock School of Public Health, National University of Singapore, Singapore. ${ }^{7}$ National Centre for Epidemiology and Population Health, Australian National University, Canberra, Australia.}

\section{Authors' contributions}

JY and VJL conceived the study, collected the data, performed the analysis, and wrote the manuscript together. CHT, ARC, JPL and BHT performed the analysis and participated in the manuscript writing. PAT participated in the manuscript writing. All authors have read and approved the final manuscript and the manuscript is currently not submitted for publication elsewhere.

\section{Competing interests}

VIL has received unrelated research support from GSK. PAT has received research support and honoraria from Baxter, Adamas, Merlion Pharma, and Novartis as well as travel support from Pfizer and Wyeth and sits on the boards of the Asia Pacific Advisory Committee on Influenza and the Asian Hygiene Council. The rest of the authors declare that we do not have any conflict of interests, financial or otherwise, in this study.

Received: 4 July 2011 Accepted: 20 January 2012

Published: 20 January 2012

\section{References}

1. Chew FT, Doraisingham S, Ling AE, Kumarasinghe G, Lee BW: Seasonal trends of viral respiratory tract infections in the tropics. Epidemiol Infect 1998, 121:121-128

2. Tang JW, Tambyah PA, Lai FY, Lee HK, Lee CK, Loh TP, Chiu L, Koay ES: Differing symptom patterns in early pandemic vs seasonal influenza infections. Arch Intern Med 2010, 170:861-867.

3. World Health Organisation: Pandemic (H1N1) 2009 - update 62 (revised 21 August 2009).[http://www.who.int/csr/don/2009_08_21/en/index.html.]
4. Chen MI, Lee VJ, Lim WY, Barr IG, Lin RT, Koh GC, Yap J, Cui L, Cook AR, Laurie K, Tan LW, Tan BH, Loh J, Shaw R, Durrant C, Chow VT, Kelso A, Chia KS, Leo YS: 2009 influenza $A(H 1 N 1)$ seroconversion rates and risk factors among distinct adult cohorts in Singapore. JAMA 2010, 303(14):1383-1391.

5. Lee VJ, Yap J, Cook AR, Tan CH, Loh JP, Koh WH, Lim EA, Liaw JC, Chew JS, Hossain I, Chan KW, Ting PJ, Ng SH, Gao Q, Kelly PM, Chen MI,

Tambyah PA, Tan BH: A clinical diagnostic model for predicting influenza among young adult military personnel with febrile respiratory illness in Singapore. PLOS ONE 2011, 6(3):e17468.

6. Cutter JL, Ang LW, Lai FY, Subramony H, Ma S, James L: Outbreak of pandemic influenza A (H1N1-2009) in Singapore, May to September 2009. Ann Acad Med Singapore 2010, 39(4):273-10

7. Wang W, Ren P, Sheng J, Mardy S, Yan H, Zhang J, Hou L, Vabret A, Buchy P, Freymuth F, Deubel V: Simultaneous detection of respiratory viruses in children with acute respiratory infection using two different multiplex reverse transcription-PCR assays. J Virol Methods 2009, 162(12):40-45.

8. Brunstein JD, Cline CL, McKinney S, Thomas E: Evidence from multiplex molecular assays for complex multipathogen interactions in acute respiratory infections. J Clin Microbiol 2008, 46(1):97-102

9. World Health Organisation: CDC protocol of realtime RT-PCR for influenza A (H1N1).[http://www.who.int/csr/resources/publications/swineflu/ realtimeptpcr/en/index.html.]

10. Hoffmann E, Stech J, Guan Y, Webster RG, Perez DR: Universal primer set for the full length amplification of all influenza A viruses. Arch Virol 2001, 146(12):2275-2289.

11. Ghedin E, Sengamalay NA, Shumway M, Zaborsky J, Feldblyum T, Subbu V, Spiro DJ, Sitz J, Koo H, Bolotov P, Dernovoy D, Tatusova T, Bao Y, St George K, Taylor J, Lipman DJ, Fraser CM, Taubenberger JK, Salzberg SL: Large-scale sequencing of human influenza reveals the dynamic nature of viral genome evolution. Nature 2005, 437(7062):1162-1166.

12. R Core Development Team: $\mathrm{R}: \mathrm{A}$ language and environment for statistical computing.[http://www.R-project.org].

13. Lee VJ, Yap J, Cook AR, Chen MI, Tay JK, Barr I, Kelso A, Tan BH, Loh JP, Lin R, Cui L, Kelly PM, Leo YS, Chia KS, Kang WL, Tambyah PA, Seet B: Effectiveness of public health measures in mitigating pandemic influenza spread: A prospective sero-epidemiological cohort study. J Infect Dis Nov 2010, 202(9):1319-1326.

14. Ong AK, Chen MI, Lin L, Tan AS, Nwe NW, Barkham T, Tay SY, Leo YS: Improving the clinical diagnosis of influenza- a comparative analysis of new influenza A (H1N1) cases. PLOS ONE 2009, 4:e8453.

15. Belongia EA, Irving SA, Waring SC, Coleman LA, Meece JK, Vandermause M, Lindstrom S, Kempf D, Shay DK: Clinical characteristics and 30-day outcomes for influenza A 2009 (H1N1), 2008-2009 (H1N1), and 20072008 (H3N2) infections. JAMA 2010, 304(10):1091-1098.

16. Carrat F, Tachet A, Rouzioux C, Housset B, Valleron AJ: Evaluation of clinical case definitions of influenza: detailed investigation of patients during the 1995-1996 epidemic in France. Clin Infect Dis 1999, 28:283-290.

Pre-publication history

The pre-publication history for this paper can be accessed here: http://www.biomedcentral.com/1471-2334/12/12/prepub

\section{doi:10.1186/1471-2334-12-12}

Cite this article as: Yap et al:: Differing clinical characteristics between influenza strains among young healthy adults in the tropics. BMC Infectious Diseases 2012 12:12. 